\title{
Leptonic B Decays from Belle
}

\section{Koji Ikado*†}

Nagoya University, Japan

E-mail: ikado@hepl.phys.nagoya-u.ac.jp

\begin{abstract}
We present a search for the decays $B^{-} \rightarrow \tau^{-} \bar{v}$ and $B^{-} \rightarrow K^{-} v \bar{v}$ in a $253 \mathrm{fb}^{-1}$ data sample collected at the $\Upsilon(4 S)$ resonance with the Belle detector at the KEKB asymmetric-energy $B$ factory. Combinatorial and continuum backgrounds are suppressed by selecting a sample of events with one fully reconstructed $B$. The decay products of the $B$ on the other side of the event are analyzed to search for $B^{-} \rightarrow \tau^{-} \bar{v}$ and $B^{-} \rightarrow K^{-} v \bar{v}$ decays. We find no significant evidence for a signal and set $90 \%$ confidence level upper limits of $\mathscr{B}\left(B^{-} \rightarrow \tau^{-} \bar{v}\right)<1.8 \times 10^{-4}$ and $\mathscr{B}\left(B^{-} \rightarrow K^{-} v \bar{v}\right)<3.6 \times 10^{-5}$.
\end{abstract}

International Europhysics Conference on High Energy Physics

July 21st - 27th 2005

Lisboa, Portugal

${ }^{*}$ Speaker.

†n behalf of the Belle collaboration 


\section{Introduction}

The purely leptonic decay $B^{-} \rightarrow \ell^{-} \bar{v}$ (charge conjugate states are implied throughout the paper) is of particular interest since it provides direct measurement of the product of the CabibboKobayashi-Maskawa(CKM) matrix element $V_{u b}$ and the $B$ meson decay constant $f_{B}$. The CKMfi tter predicts the $B^{-} \rightarrow \tau^{-} \bar{v}$ branching fraction to be $\left(9.3_{-2.3}^{+3.4}\right) \times 10^{-5}$ [1]. No evidence for an enhancement relative to the Standard Model(SM) prediction was observed in previous experimental studies. The most stringent upper limit has been achieved by the BABAR Collaboration $: \mathscr{B}\left(B^{-} \rightarrow \tau^{-} \bar{v}\right)<4.2 \times 10^{-4}$ at $90 \%$ confi dence level (C.L.) [2].

Flavor-changing neutral-current transition such as $b \rightarrow s v \bar{v}$ occurs in the SM via one-loop box or electroweak penguin diagrams with heavy particles in the loops. The SM $B^{-} \rightarrow K^{-} v \bar{v}$ branching fraction has been estimated to be $\left(3.8_{-0.6}^{+1.2}\right) \times 10^{-6}[3,4]$, while the most stringent published experimental limit is $\mathscr{B}\left(B^{-} \rightarrow K^{-} v \bar{v}\right)<5.2 \times 10^{-5}$ at $90 \%$ C.L. [5]

\section{Data Sample}

We use a $253 \mathrm{fb}^{-1}$ data sample containing $275 \times 10^{6} \mathrm{~B}$ meson pairs collected with the Belle detector at the KEKB asymmetric-energy $e^{+} e^{-}(3.5$ on $8 \mathrm{GeV})$ collider [6] operating at the $\Upsilon(4 S)$ resonance $(\sqrt{s}=10.58 \mathrm{GeV})$. The strategy adopted for this analysis is to reconstruct exclusively the decay of one of the $B$ mesons in the event and compare properties of the remaining particle(s) in the event (referred to as the signal side) to those expected for signal and background. All the tracks and photon candidates in the event not used to reconstruct the $B$ are studied to search for $B^{-} \rightarrow \tau^{-} \bar{v}$ and $B^{-} \rightarrow K^{-} v \bar{v}$. Fully reconstructed $B$ mesons, $B_{\text {rec }}$, are observed in the following decay modes: $B^{+} \rightarrow \bar{D}^{(*) 0} \pi^{+}, \bar{D}^{(*) 0} \rho^{+}, \bar{D}^{(*) 0} a_{1}^{+}$and $\bar{D}^{(*) 0} D_{S}^{(*)+} . \bar{D}^{0}$ candidates are reconstructed as $\bar{D}^{0} \rightarrow K^{+} \pi^{-}$, $K^{+} \pi^{-} \pi^{0}, K^{+} \pi^{-} \pi^{+} \pi^{-}, K_{s}^{0} \pi^{0}, K_{s}^{0} \pi^{-} \pi^{+}, K_{s}^{0} \pi^{-} \pi^{+} \pi^{0}$ and $K^{-} K^{+} . \bar{D}^{* 0}$ mesons are reconstructed by combining the $\bar{D}^{0}$ candidates with a pion or a photon. $D_{S}^{+}$candidates are reconstructed in the decay modes $D_{S}^{+} \rightarrow K_{S}^{0} K^{+}$and $K^{+} K^{-} \pi^{+}$, and $D_{S}^{*+}$ mesons are reconstructed by combining the $D_{S}^{+}$ candidates with a photon. We obtain $(4.00 \pm 0.24) \times 10^{5}$ tagged events $\left(N_{B^{+} B^{-}}\right)$with the purity of 0.55 , where the uncertainty on $N_{B^{+} B^{-}}$is dominated by systematic errors.

\section{Event Selection}

In the events where a $B_{\text {rec }}$ is reconstructed, we search for decays into a $\tau$ plus a neutrino and a $K$ plus two neutrinos. Candidate events are required to have one or three signal-side charged track(s) with the total charge being opposite to that of the reconstructed $B$. The $\tau$ lepton is identifi ed in the following decay channels: $\tau^{-} \rightarrow \mu^{-} v \bar{v}, \tau^{-} \rightarrow e^{-} v \bar{v}, \tau^{-} \rightarrow \pi^{-} v, \tau^{-} \rightarrow \pi^{-} \pi^{0} v$, and $\tau^{-} \rightarrow$ $\pi^{-} \pi^{+} \pi^{-} \nu$. We require the charged particles to be identifi ed as leptons, pions or kaons. The event is required to have zero net charge and $E_{\mathrm{ECL}}$ less than $0.3 \mathrm{GeV}$ where $E_{\mathrm{ECL}}$ is the remaining energy calculated by adding the energy of the photons that are not associated with either the $B_{\text {rec }}$ or the $\pi^{0}$ candidate from $\tau^{-} \rightarrow \pi^{-} \pi^{0} v$ decay. For all modes except the $\tau^{-} \rightarrow \pi^{-} \pi^{0} v$ mode we reject events with $\pi^{0}$ mesons in the recoil against $B_{\text {rec }}$. We place the following requirements on the center-ofmass (CM) momentum of the track(s), $p_{\pi^{-}}>0.8 \mathrm{GeV} / c$ for $\tau^{-} \rightarrow \pi^{-} v, p_{\pi^{-} \pi^{0}}>1.2 \mathrm{GeV} / c$ for $\tau^{-} \rightarrow \pi^{-} \pi^{0} v, p_{\pi^{-} \pi^{+} \pi^{-}}>1.4 \mathrm{GeV} / c$ for $\tau^{-} \rightarrow \pi^{-} \pi^{+} \pi^{-} v$, and $p_{K^{-}}>1.2 \mathrm{GeV} / c$ for $B^{-} \rightarrow K^{-} v \bar{v}$. 
The event is required to have the total missing momentum greater than $0.2 \mathrm{GeV} / c$ for all modes except leptonic decay modes and the direction of missing momentum $-0.86<\cos \theta_{\text {miss }}^{*}<0.95$ in the $\mathrm{CM}$ frame. Further requirements are made on the invariant mass of two or three pions $\left|M_{\pi \pi}-M_{\rho}\right|<0.15 \mathrm{GeV} / c^{2}$ and $\left|M_{\pi \pi \pi}-M_{a_{1}^{+}}\right|<0.2 \mathrm{GeV} / c^{2}$. The selection effi ciencies for each decay mode we consider in this analysis are determined from a large sample of GEANT-based Monte Carlo simulations [8] for $B^{-} \rightarrow \tau^{-} \bar{v}$ and $B^{-} \rightarrow K^{-} v \bar{v}$ events generated by EvtGen decay package[9]. We compute the effi ciency as the ratio of the number of events surviving each of our selections over the number of fully reconstructed $B^{ \pm}$.

\section{Background Estimation}

The most powerful variable for separating signal and background is the remaining energy $E_{\mathrm{ECL}}$. We use different energy cuts for neutral clusters contributing to $E_{\mathrm{ECL}}$ for the barrel part and end-cap parts since the effect of beam background is severe in the end-caps. For signal events the neutral clusters contributing to $E_{\mathrm{ECL}}$ can only come from beam background, therefore the signal events peak at low $E_{\mathrm{ECL}}$ and the background events, which contain additional sources of neutral clusters, are distributed toward higher $E_{\mathrm{ECL}}$ values. The $E_{\mathrm{ECL}}<0.3 \mathrm{GeV}$ region is defi ned as the signal region and the $0.45<E_{\mathrm{ECL}}<1.5 \mathrm{GeV}$ region is defi ned as the sideband region. The $E_{\mathrm{ECL}}$ shape in the MC distribution is used to extrapolate the sideband data to the signal region. We calcurate the ratio of the number of MC events in the signal region to that in the sideband one, $r_{M C}$. Using the number of data events in the sideband and the ratio $r_{M C}$, the number of expected background events in the signal region is estimated.

\section{Results}

After fi nalizing the signal selection criteria, the signal region $\left(E_{\mathrm{ECL}}<0.3 \mathrm{GeV}\right)$ in the onresonance data is examined. Table 1 lists the number of observed events in data in the signal region, together with the expected number of signal and background events in the signal region. Since we do not observe signifi cant excess over the expected background, we set upper limits on the branching fraction for $\mathscr{B}\left(B^{-} \rightarrow \tau^{-} \bar{v}\right)$ and $\mathscr{B}\left(B^{-} \rightarrow K^{-} v \bar{v}\right)$. To extract the upper limits, we fi $t$ the observed $E_{\mathrm{ECL}}$ distributions to the expected background and signal, using maximum likelihood method. The negative log likelihood function is minimized using MINUIT [10] with respect to $n_{b}$ for each $n_{s}\left(=\varepsilon_{i} \cdot N_{B^{+} B^{-}} \cdot \mathscr{B}\right)$. The $90 \%$ C. L. upper limit on the branching fraction $\mathscr{B}$ is calculated by $0.9=\frac{\int_{0}^{\mathscr{B}} 90 \mathscr{L}(\mathscr{B}) d \mathscr{B}}{\int_{0}^{\infty} \mathscr{L}(\mathscr{B}) d \mathscr{B}}$. For $B^{-} \rightarrow \tau^{-} \bar{v}$, we calculate the likelihood function for each different decay mode $\left(\mathscr{L}_{i}(\mathscr{B})\right)$. The total likelihood function is defi ned by $\mathscr{L}(\mathscr{B})=\prod_{i=1}^{n_{c h}} \mathscr{L}_{i}(\mathscr{B})$ where $n_{c h}$ is the number of decay modes for $B^{-} \rightarrow \tau^{-} \bar{v}$. We convolve the systematic uncertainty into the likelihood function, $\mathscr{L}(\mathscr{B})$, by replacing each point of $\mathscr{L}(\mathscr{B})$ by a Gaussian distribution centered at that point with a width $\triangle \mathscr{B}$ which is determined from systematic uncertainty study. We obtain upper limits on the branching fraction at the $90 \%$ C.L. of

$$
\begin{gathered}
\mathscr{B}\left(B^{-} \rightarrow \tau^{-} \bar{v}\right)<1.8 \times 10^{-4} \\
\mathscr{B}\left(B^{-} \rightarrow K^{-} v \bar{v}\right)<3.6 \times 10^{-5}
\end{gathered}
$$




\begin{tabular}{c|c|cccc}
\hline \multicolumn{2}{c|}{ Decay Mode } & $\begin{array}{c}\text { Signal } \\
\text { Effi ciency }(\%)\end{array}$ & $\begin{array}{c}\text { Signal } \\
\text { Expected }\end{array}$ & $\begin{array}{c}\text { Background } \\
\text { Expected }\end{array}$ & $\begin{array}{c}\text { Observed } \\
\text { Events }\end{array}$ \\
\hline \hline \multirow{5}{*}{$B^{-} \rightarrow \tau^{-} \bar{v}$} & $\tau^{-} \rightarrow \mu^{-} v \bar{v}$ & $9.8 \pm 0.1$ & $3.9 \pm 0.1$ & $11.8 \pm 3.3$ & 8 \\
& $\tau^{-} \rightarrow e^{-} v \bar{v}$ & $9.4 \pm 0.1$ & $3.8 \pm 0.1$ & $9.5 \pm 3.0$ & 10 \\
& $\tau^{-} \rightarrow \pi^{-} v$ & $8.4 \pm 0.1$ & $3.4 \pm 0.1$ & $3.5 \pm 1.7$ & 11 \\
& $\tau^{-} \rightarrow \pi^{-} \pi^{0} v$ & $3.5 \pm 0.1$ & $1.4 \pm 0.1$ & $3.0 \pm 1.6$ & 4 \\
& $\tau^{-} \rightarrow \pi^{-} \pi^{+} \pi^{-} v$ & $2.6 \pm 0.1$ & $1.0 \pm 0.1$ & $3.6 \pm 1.7$ & 6 \\
\hline \hline \multicolumn{2}{c|}{ Total } & $33.7 \pm 0.2$ & $13.5 \pm 0.2$ & $31.4 \pm 5.3$ & 39 \\
\hline \multicolumn{2}{c|}{$B^{-} \rightarrow K^{-} v \bar{v}$} & $42.8 \pm 1.8$ & $0.70 \pm 0.03$ & $2.6 \pm 1.6$ & 4 \\
\hline
\end{tabular}

Table 1: Number of observed data events in the signal region, together with the number of expected signal events and background events. Errors are the statistical only. The numbers of expected signal are obtained by assuming that $\mathscr{B}\left(B^{-} \rightarrow \tau^{-} \bar{v}\right)=10^{-4}$ and $\mathscr{B}\left(B^{-} \rightarrow K^{-} v \bar{v}\right)=4 \times 10^{-6}$.

which are the most stringent upper limits on these processes to date.

We thank the KEKB group for the excellent operation of the accelerator, the KEK cryogenics group for the effi cient operation of the solenoid, and the KEK computer group and the NII for valuable computing and Super-SINET network support. We acknowledge support from MEXT and JSPS (Japan); ARC and DEST (Australia); NSFC (contract No. 10175071, China); DST (India); the BK21 program of MOEHRD and the CHEP SRC program of KOSEF (Korea); KBN (contract No. 2P03B 01324, Poland); MIST (Russia); MHEST (Slovenia); SNSF (Switzerland); NSC and MOE (Taiwan); and DOE (USA).

\section{References}

[1] J. Charles et al. (CKMfitter Group), Eur. Phys. J. C 41, 1 (2005) and the updated results presented at CKM2005 workshop.

[2] B. Aubert (BABAR Collaboration), Phys. Rev. Lett. 95, 041804 (2005).

[3] A. Faessler, T. Gutsche, M. A. Ivanov, J. G. Korner and V. E. Lyubovitskij, Eur. Phys. J. directC 4, 18 (2002).

[4] G. Buchalla, G. Hiller and G. Isidori, Phys. Rev. D 63, 014015 (2001).

[5] B. Aubert et al. (BABAR Collaboration), Phys. Rev. Lett. 94, 101801 (2005).

[6] S. Kurokawa and E. Kikutani, Nucl. Instrum. Methods Phys. Res., Sect. A 499, 1 (2003).

[7] Belle Collaboration, A. Abashian et al. Nucl. Instrum. Methods Phys. Res., Sect. A 479, 117 (2002).

[8] R. Brun et al., GEANT3.21, CERN Report DD/EE/84-1 (1984).

[9] See the EvtGen package home page, http://www.slac.stanford.edu/ lange/EvtGen/.

[10] F. James and M. Roos, Comput. Phys. Commun. 10 (1975) 343. 